\title{
School milk scheme pilot in preschools
}

\author{
E. Greene and C. Murrin \\ School of Public Health, Physiotherapy and Sports Science, UCD Belfield, Dublin 4
}

As childhood is a crucial period for optimisation of peak bone mass, adequate calcium intake is essential. Dairy products, such as milk and yoghurt provide $35 \%$ of Irish children's calcium intake. However, milk consumption among Irish children has reduced in the past decade $^{(1)}$. Furthermore, it is known that food preference is an important influence on food intake. Preferences are acquired in early life and can continue into adulthood ${ }^{(2)}$. Therefore intervening before school age, may be effective in promoting a preference for milk which may persist further into childhood and adolescence. The EU School Milk Scheme (SMS) promotes milk consumption among schoolchildren by providing subsidised milk to schools. It has been operating in Ireland since 1982, predominantly in primary schools $^{(3)}$

The aim of this research is to evaluate the feasibility and effectiveness of the SMS in preschools, which aims to promote a liking for milk among preschool-aged children and to improve attitudes towards milk among preschool staff and parents. The current objective is to describe the existing attitudes towards milk among preschool staff prior to delivery of the SMS.

The SMS preschool pilot was carried out among 3-5 year-old children in twelve Irish preschools, over a three-week period. The pilot involved provision of milk for daily distribution to children and the delivery of the 'Moo Crew' educational package by preschool staff, aimed to improve children's knowledge of the origin of milk and its benefits. Preschool staff members completed a baseline questionnaire, collecting data on preschool characteristics and attitudes towards milk. Questionnaires were analysed using descriptive statistics.

There was a mean (SD) of 40 (12) 3-5 year-old children in each preschool $(n=11)$, of which $45 \%$ attended preschool full-time and $55 \%$ part-time. Services served an average of three meals and snacks per day, with all preschools serving at least one meal or snack. Milk was served regularly in $4(36 \%)$ preschools and occasionally in $3(27 \%)$, all of which reported serving full-fat milk. All services reported having a healthy eating policy, however guidelines for serving milk were included in just five preschool policies. Eighty percent of staff members reported liking the taste of milk. Staff felt that milk consumption was important to them and agreed that milk has benefits for adults (82\%) and for children (91\%). Six respondents listed factors related to bone health and dental health as health benefits associated with drinking milk.

Preschool staff have a positive attitude towards milk and are aware of its nutritional benefits Therefore, preschool services may be an effective setting in which to promote milk consumption among 3-5 year-old children in Ireland, as they serve a minimum of one meal or snack to children per day.

1. National Children's Food Survey II: Summary Report. Irish Universities Nutrition Alliance (2019)

2. De Cosmi V, Scaglioni S, Agostoni C. (2017) Nutrients, 9, 2.

3. School Milk Scheme (SMS) Department of Agriculture, Food and the Marine [Available at: https://www.agriculture.gov.ie/customerservice/ euschoolscheme/schoolmilkschemesms/]. 